\title{
Entrainment transition in populations of random frequency oscillators
}

\author{
Hyunsuk Hong, ${ }^{1}$ Hugues Chaté, ${ }^{2}$ Hyunggyu Park, ${ }^{3}$ and Lei-Han Tang ${ }^{4}$ \\ ${ }^{1}$ Department of Physics and RINPAC, Chonbuk National University, Jeonju 561-756, Korea \\ ${ }^{2}$ Service de Physique de l'Etat Condensé, CEA-Saclay, 91191 Gif-sur-Yvette, France \\ ${ }^{3}$ School of Physics, Korea Institute for Advanced Study, Seoul 130-722, Korea \\ ${ }^{4}$ Department of Physics, Hong Kong Baptist University, Kowloon Tong, Hong Kong SAR, China
}

(Dated: October 19, 2018)

\begin{abstract}
The entrainment transition of coupled random frequency oscillators is revisited. The Kuramoto model (global coupling) is shown to exhibit unusual sample-dependent finite size effects leading to a correlation size exponent $\bar{\nu}=5 / 2$. Simulations of locally-coupled oscillators in $d$ dimensions reveal two types of frequency entrainment: mean-field behavior at $d>4$, and aggregation of compact synchronized domains in three and four dimensions. In the latter case, scaling arguments yield a correlation length exponent $\nu=2 /(d-2)$, in good agreement with numerical results.
\end{abstract}

PACS numbers: 05.45.Xt, 05.45.-a, 89.75.-k

Collective oscillations are abundant in physical, chemical, and biological systems far from equilibrium [1, 2, $2,3,4$, 5]. Such synchronized behavior has been widely explored via various systems of coupled oscillators [6, 7, 8, 9]. Although several theoretical methods are available to treat systems of identical oscillators with or without noise [10, 11, 12], the description of synchronization in coupled oscillators with a broad distribution of intrinsic frequencies is still a largely unsolved problem [8, 9]. More recently, the study of dynamics on complex networks, including the human brain, brought renewed interest into the subject [13].

The onset of system-wide synchronization at some coupling strength is often compared to critical phenomena in equilibrium systems, where scaling concepts provide an adequate theoretical basis for quantitative analysis. The scaling approach has been successfully applied to the Kuramoto model [6], but its extension to finitedimensional systems has stumbled over a number of difficulties [14, 15, 16, 17]. Even in the globally-coupled case, the critical size needed to maintain a coherent group of synchronized oscillators in a large but finite population, as well as their ensuing dynamics, have not been fully understood [8, 9, 17]. In $d$-dimensional systems with local coupling, $d=2$ is generally accepted as the lower critical dimension for macroscopic entrainment, but the upper critical dimension is uncertain due to peculiar size effects seen in numerical investigations 17.

In this Letter, we re-examine the above issues critically and establish two types of behavior at the onset of macroscopic synchronization in coupled random frequency oscillators. In the globally coupled case and in finite dimensions $d>4$, we show that frequency entrainment is accompanied by spontaneous symmetry breaking in the phase, and as such shares many features with the usual ordering transition in equilibrium systems with $O(2)$ symmetry. The correlation size exponent $\bar{\nu}$ governing finite-size scaling at criticality, however, takes on the value $\frac{5}{2}$ in the present case rather than 2 in usual mean-field theories [18]. For $d \leq 4$, the above type of symmetry-breaking is ruled out by diverging phase fluctuations. Instead, oscillators are first entrained with their neighbors to form compact, synchronized domains which grow upon further increase in coupling. System-wide entrainment is achieved only when the largest domain reaches system size. This gives rise to size- and sampledependent thresholds whose behavior is quantified.

We begin with the globally-coupled Kuramoto model defined by the following set of dynamical equations:

$$
\frac{d \phi_{j}}{d t}=\omega_{j}-K \Delta \sin \left(\phi_{j}-\theta\right)
$$

Here $\phi_{j}$ is the phase of the $j$ th oscillator $(j=1, \ldots, N)$, and $\omega_{j}$ its natural frequency, drawn from a normalized distribution $g(\omega)$. To be definite, here we take $g(\omega)$ to be Gaussian with zero mean and unit variance. The parameter $K$ sets the strength of coupling to the global quantities $\Delta$ and $\theta$ defined via

$$
\Delta e^{i \theta}=N^{-1} \sum_{j=1}^{N} e^{i \phi_{j}} .
$$

In the classic work by Kuramoto, the existence of an entrained state is established by considering solutions to (11) at a constant $\Delta$. After an initial transient, oscillators with $\left|\omega_{j}\right|<K \Delta$ reach a fixed angle $\phi_{j}^{(0)}$ while those with $\left|\omega_{j}\right|>K \Delta$ are in a running pendulum state. In the limit $N \rightarrow \infty$, the self-consistent equation for $\Delta$ reads

$$
\Delta=\int_{-K \Delta}^{K \Delta} d \omega g(\omega) \sqrt{1-\left(\frac{\omega}{K \Delta}\right)^{2}} \equiv \Psi(\Delta),
$$

which has a nontrivial solution when $K>K_{\mathrm{c}}=\frac{2}{\pi g(0)}$. For a population of finite size, $\Psi(\Delta)$ is replaced by

$$
\tilde{\Psi}(\Delta) \equiv \frac{1}{N} \sum_{j,\left|\omega_{j}\right|<K \Delta} \sqrt{1-\left(\frac{\omega_{j}}{K \Delta}\right)^{2}},
$$


which contains a sample-dependent correction $\delta \tilde{\Psi} \equiv \tilde{\Psi}-$ $\langle\tilde{\Psi}\rangle=\tilde{\Psi}-\Psi \propto \sqrt{N_{\mathrm{s}}} / N$. Here $N_{\mathrm{s}}$ is the number of oscillators in the frequency interval $(-K \Delta, K \Delta)$, and $\langle\cdot\rangle$ denotes sample average. Close to the transition, the selfconsistency equation $\Delta=\tilde{\Psi}(\Delta)$ can be written as

$$
\Delta=a K \Delta-c(K \Delta)^{3}+\delta \tilde{\Psi},
$$

where $a=K_{\mathrm{c}}^{-1}$ and $c=-\pi g^{\prime \prime}(0) / 16$. The variance of $\delta \tilde{\Psi}$ is given by $\left\langle(\delta \tilde{\Psi})^{2}\right\rangle=\frac{4}{3} g(0) K \Delta / N+O\left(\Delta^{2} / N\right)$. Hence the solution to Eq. (5) takes the scaling form

$$
\Delta(K, N)=N^{-1 / 5} f\left(k N^{2 / 5}\right),
$$

where $k \equiv\left(K-K_{\mathrm{c}}\right) / K_{\mathrm{c}}$. The scaling function $f(x)$ is sample-dependent and satisfies the equation,

$$
x f-c K_{\mathrm{c}}^{3} f^{3}+(8 / 3 \pi)^{1 / 2} \epsilon f^{1 / 2}=0 .
$$

Here $\epsilon \equiv \delta \tilde{\Psi} /\left\langle(\delta \tilde{\Psi})^{2}\right\rangle^{1 / 2}$ is a Gaussian random variable with zero mean and unit variance.

Equation (6) resembles the finite-size scaling form in various mean-field models of equilibrium phase transitions [18], but the value of the exponent $\bar{\nu}=\frac{5}{2}$ is quite unusual. To check that $\Delta$ in the transition region is dominated by $\delta \tilde{\Psi}$ arising from "density fluctuations" of oscillators along the frequency axis, we have performed extensive simulations of Eq. (11). Figure 1(a) illustrates sample-to-sample fluctuations in the time-averaged value of $\Delta^{2}$. The onset of entrainment spreads over a distance $\delta K \sim N^{-2 / 5}$ around the nominal $K_{\mathrm{c}}$, as seen from the scaling plot of $\left\langle\Delta^{2}\right\rangle$ against $K$ at various sizes $N$ in Fig. 1(b). The dashed line there is obtained by averaging solutions to Eq. (17) at many different values of $\epsilon$ drawn from a Gaussian distribution. The agreement between the predicted scaling and simulation data is satisfactory on the entrained side and sufficiently close to the transition, on which Eq. (5) is based. Figure1 (c) shows the relative strength of temporal fluctuations $\delta \Delta(t) \equiv \Delta(t)-\bar{\Delta}$ of the order parameter in the transition region. (Here the overline bar denotes time average.) On the detrained side, the ratio $\left\langle\overline{\delta \Delta^{2}}\right\rangle /\left\langle\bar{\Delta}^{2}\right\rangle$ at large $N$ approaches the value $\frac{4}{\pi}-1$ for Gaussian fluctuations. On the entrained side, this quantity decreases as $N^{-1}$ as expected from independent fluctuations. At $K=K_{c}$, the data clearly decrease with $N$, lending further support to our analysis.

We now turn to locally-coupled oscillators on $d$ dimensional hypercubic lattices, for which the dynamical equations take the form

$$
\frac{d \phi_{i}}{d t}=\omega_{i}-K \sum_{j \in \Lambda_{i}} \sin \left(\phi_{i}-\phi_{j}\right) .
$$

where $\Lambda_{i}$ is the set of all nearest neighbors of site $i$.

At sufficiently large $K$, Eq. (8) can be treated using a linear approximation [14, 17]. In this limit, the system enters a "fully entrained" state where the random term
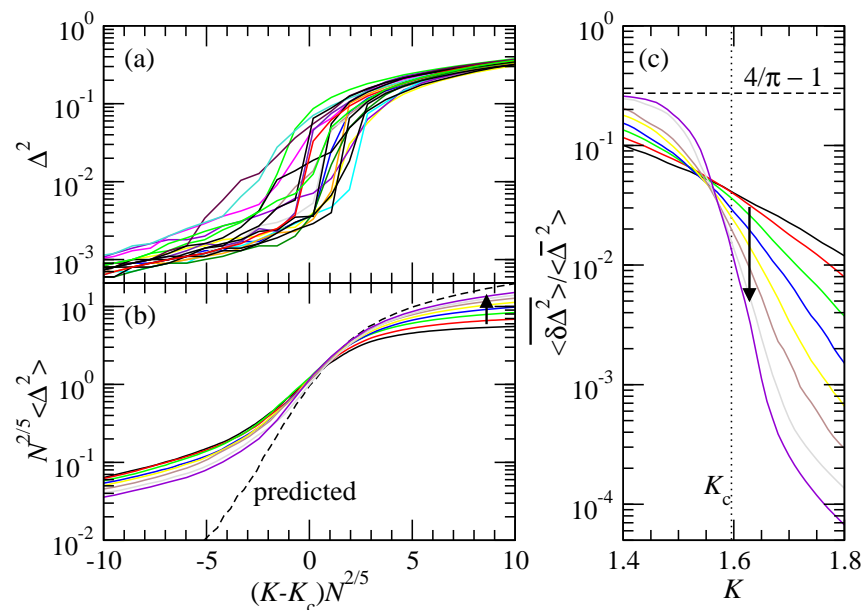

FIG. 1: (Color online) $\Delta^{2}$ against $K$ for globally-coupled oscillators. (a) Time-averaged value of $\Delta^{2}$ for 20 different samples $(N=12800)$. (b) Scaling plot for $N=$ $100,200, \ldots, 12800$, in increasing order as indicted by the arrow (averaged over 100 samples for each $N$ ). Dashed line: see text. (c) Relative strength of temporal versus sample-tosample fluctuations of $\Delta$ for a set of system sizes as in (b).

$\omega_{i}$ is balanced by local gradients of a static phase field $\phi_{i}^{(0)}$. Salient features of this state are summarized as follows: i) For $d>4, \phi_{i}^{(0)}$ has bounded variations even when the linear system size $L \rightarrow \infty$. Consequently, the entrained state has a global phase that breaks the $O(2)$ symmetry. ii) For $2<d \leq 4$, variations in $\phi_{i}^{(0)}$ grow as $L^{(4-d) / 2}$. Hence an entrained state cannot be assigned a definite phase. iii) For $d \leq 2$, local phase gradients have an infra-red divergence $L^{\overline{(2}-d) / 2}$. In this case, the system is detrained beyond a coherence length $\xi \sim K^{2 /(2-d)}$.

As $K$ decreases, the local phase gradients need to increase to counter the $\omega_{i}$ 's but there is a limit to how far this can go in the Kuramoto model. Upon proliferation of phase slips and runaway oscillators, two scenarios can be contemplated for the destruction of global entrainment: i) Oscillators break away individually from the entrained group until the latter is reduced to an infinitesimal fraction of the system as in the globally-coupled model. ii) A more collective form of phase slips takes place along "domain boundaries" that break the system into locally synchronized clusters, starting from the largest scale. For ii) to preempt i), there must be pre-existing large phase differences (i.e., "strain") across the system, which is the case in the entrained state at and below $d=4$ but not above. This suggests that the nature of the detrainment transition is quite different above and below $d=4$.

To characterize system-wide coherent phase motion in the entrained state, we introduce the Edwards-Anderson order parameter

$$
\Delta_{\mathrm{EA}}=\lim _{t-t_{0} \rightarrow \infty} \frac{1}{N}\left|\sum_{j} e^{i\left[\phi_{j}(t)-\phi_{j}\left(t_{0}\right)\right]}\right| .
$$


Compared to $\Delta$ defined by Eq. (2), $\Delta_{\mathrm{EA}}$ is incensitive to the static phase deformation discussed above, and hence can also be used for $d \leq 4$.

We have carried out extensive numerical simulations of (8) to investigate the two types of transition behavior and associated critical properties and finite-size effects. Periodic boundary conditions are used. Let us first examine the spatio-temporal behavior of the phase advance $\Delta \phi_{i}=\phi_{i}(t+T)-\phi_{i}(t)$ of oscillators over a sufficiently long interval $T$ in a given oscillator population near the entrainment transition. Figure 2 (a) presents the distribution $P(v)$ of the mean phase velocity $v_{i}=\Delta \phi_{i} / T$ over an interval $T=10000$ for a $N=16^{5}$ system in $d=5$ dimensions. The inset shows a magnified plot of the peak region where values of $\Delta \phi$ within each $2 \pi$ interval are resolved. Evidently, entrainment here is accompanied by the selection of a global phase, i.e., the transition is of the symmetry-breaking type. Comparing the two distributions at $K=0.2$ and $K=0.205$, we see that only a small fraction of oscillators participate in the onset of entrainment, while the rest are not much affected at this stage. This is very similar to the behavior of the globallycoupled case. At $K=0.205$, the wings can be fitted well to a weak, integrable power-law, $P(v) \sim|v|^{-1 / 2}$.

Figure 2(b) shows $P(v)$ for a $d=3$ system of $N=64^{3}$ oscillators at three different values of $K$ around the entrainment transition. Here $T=5000$. A strong narrowing of $P(v)$ is seen in the entire critical region, indicating the formation of large synchronized domains well below global entrainment. The wings fall off roughly as $v^{-2}$. Consequently, most oscillators are moving at phase velocities close to that of the peak. However, as seen in the inset, the actual phase advance $\Delta \phi_{i}$ of these oscillators is much less entrained as compared to the $d=5$ case.

Further indication of entrainment through growth and aggregation of locally synchronized domains is found in the spatial structures of the $\Delta \phi_{i}$ 's, as depicted in Fig. 3 for one layer of the $d=3$ system. At $K=0.66$, a large, spanning domain of entrained oscillators coexist with smaller clusters of oscillators at varying phase velocities. When $K$ is decreased to 0.64 , part of this largest domain undergoes a phase slip over the time period, as indicated by the arrow in Fig. 3(b). This process of detrainment through phase slips continues down to the smallest scale upon further weakening of the coupling.

The actual dynamics of phase slip initiation and propagation, particularly in the presence of runaway oscillators, is rather complicated and will be left for future investigation. It is, however, possible to gain some intuition about the factors governing the typical domain size through the following consideration. In Fig. 3(b), for example, each of the light-colored regions can be considered as critical in the sense that a weaker $K$ would lead to fragmentation of the domain while a stronger $K$ would lead to merging with its neighbors. Let $\delta K$ be the increment in $K$ needed for the latter process to

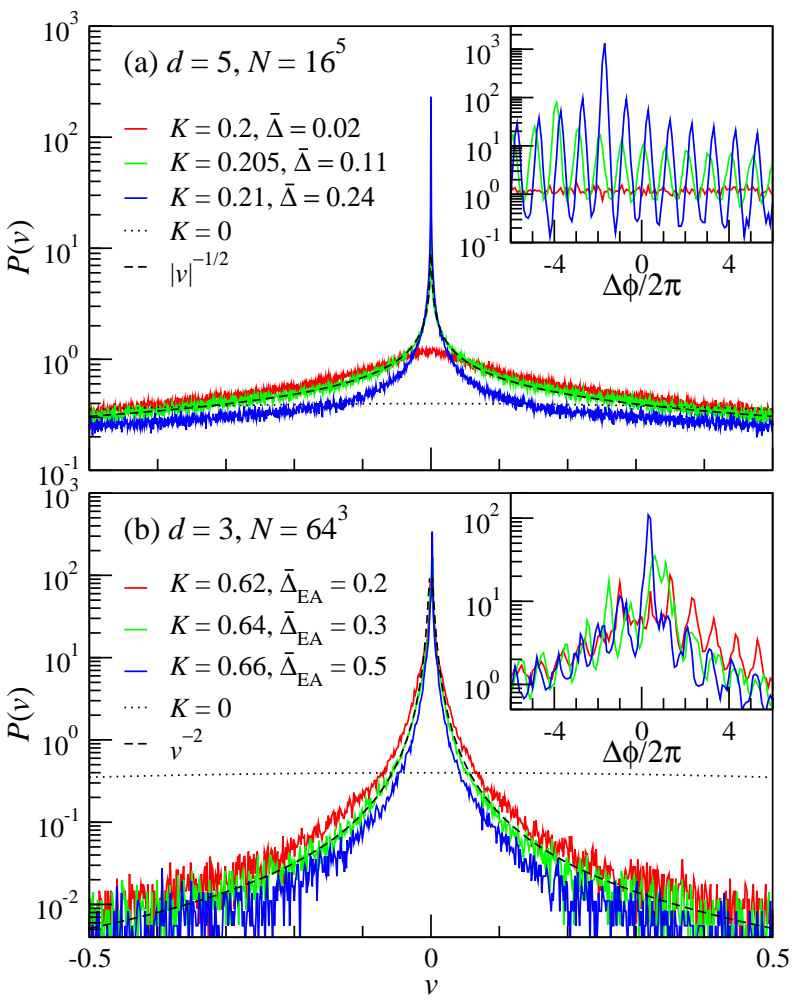

FIG. 2: (Color online) Distribution of phase velocities at various values of the coupling strength $K$ in (a) five, (b) three dimensions. Insets show the peak region in detail. The timeaveraged value of the order parameter are listed. (a) $\mathrm{K}=0.62$

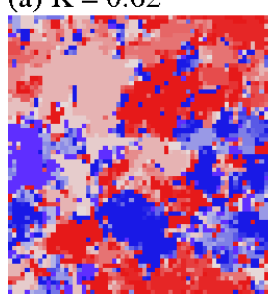

(b) $\mathrm{K}=0.64$

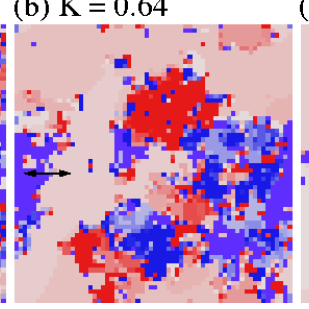

(c) $\mathrm{K}=0.66$

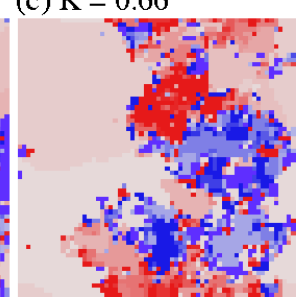

FIG. 3: (Color online) Spatial structure of $\phi_{i}(t+T)-\phi_{i}(t)$ in one layer from the sample depicted in Fig. 2(b). Here $T=5000$. Color scale from blue: $-12 \pi$ to red: $12 \pi$.

occur when the typical size of light-colored domains is $\xi$. Upon synchronization of two neighboring domains, their phase difference $\Delta \phi_{0} \sim \xi^{(4-d) / 2}$ needs to be accommodated. This is particularly so at the slip boundary, where bonds are turned from being barely unstable to barely stable. The strength of these bonds is of order $\delta K$, which offsets the phase gradient $\Delta \phi_{0} / \xi \sim \xi^{(2-d) / 2}$. Let $K_{c}$ be the value of $K$ when $\xi=\infty$, the above analysis yields a prediction for the size of synchronized domains $\xi \sim \delta K^{-\nu} \simeq\left(K_{\mathrm{c}}-K\right)^{-\nu}$ at a given $K$, where $\nu=\frac{2}{d-2}$.

Figure 4 shows the entrainment order parameter against $K$ for various system sizes. Thirty to several hundred samples were used to obtain the average in each 

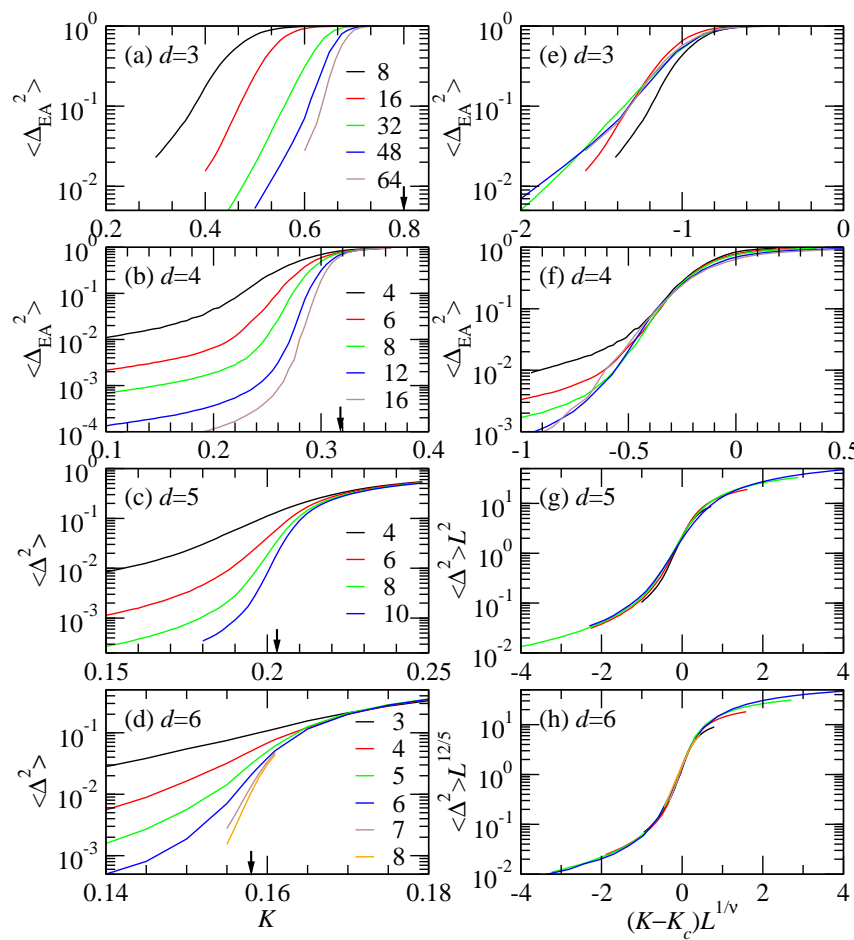

FIG. 4: (Color online) Order parameter against $K$ for various sizes ( $L$ values given on each graph) in three to six dimensions. Values of $K_{\mathrm{c}}$ [indicated by arrows in (a)-(d)] and $\nu$ used in the scaling plots are (e) $K_{\mathrm{c}}=0.8, \nu=2$; (f) $K_{\mathrm{c}}=0.318, \nu=1$; (g) $K_{\mathrm{c}}=0.203, \nu=\frac{1}{2}$; (h) $K_{\mathrm{c}}=0.158, \nu=\frac{5}{12}$.

case. To verify the scaling predictions given above, we have analyzed the data with the help of the usual finitesize scaling ansatz,

$$
\left\langle\Delta^{2}\right\rangle=L^{-2 \beta / \nu} \Phi\left(k L^{1 / \nu}\right),
$$

where $k=\left(K-K_{\mathrm{c}}\right) / K_{\mathrm{c}}$ and $\beta$ and $\nu$ are the order parameter and correlation length exponents, respectively. For $d=5$ and 6 , excellent data collapse is achieved using $\beta=\frac{1}{2}$ and $\nu=\frac{\bar{\nu}}{d}=\frac{5}{2 d}$ as in the globally-coupled case. Interestingly, the scaling extends well into the detrained phase. Noting that $\chi=N\left\langle\Delta^{2}\right\rangle$ corresponds to the susceptibility in equilibrium magnetic systems, we conclude that the exponent $\gamma=d \nu-2 \beta=\frac{3}{2}$ for $d>4$ and differs from the globally coupled case [19].

For $d=3$ and 4 , there is no convergence with increasing system size (left panel) until $\left\langle\Delta_{\mathrm{EA}}^{2}\right\rangle$ reaches a value near one. This behavior is consistent with the idea that global entrainment takes place only when locally synchronized domains join to span the whole system. Overall, a good data collapse is achieved in each case using the predicted exponents $\beta=0$ and $\nu=\frac{2}{d-2}$.

In summary, through analytical arguments and large scale simulations of the Kuramoto model in finite dimensions, we have established two types of critical behavior at the onset of global entrainment. Above four dimensions, entrainment breaks the global phase symmetry, as in the globally-coupled model, with identical scaling exponents $\beta=\frac{1}{2}$ and $\bar{\nu}=\frac{5}{2}$. For $2<d \leq 4$, and in particular for the physical dimension $d=3$, global entrainment (detrainment) occurs via the aggregation (fragmentation) of synchronized domains. The size of such domains obeys scaling with an exponent $\nu=\frac{2}{d-2}$.

This work was supported by the Korea Research Foundation Grant funded by the Korean Government (MOEHRD) (KRF-2006-331-C00123) (HH), by the Research Grants Council of the HKSAR (2017/03P and HKU3/05C) and by the Hong Kong Baptist University (FRG/01-02/II-65) (LHT).

[1] A. T. Winfree, The Geometry of Biological Time (Springer-Verlag, New York, 1980); J. Theor. Biol. 16, 15 (1967).

[2] Y. Kuramoto, in Proceedings of the International Symposium on Mathematical Problems in Theoretical Physics, edited by H. Araki (Springer-Verlag, New York, 1975); Chemical Oscillations, Waves, and Turbulence (SpringerVerlag, Berlin, 1984).

[3] A. S. Pikovsky, M. Rosenblum, and J. Kurths, Synchronization: A Universal Concept in Nonlinear Science (Cambridge University Press, Cambridge, 2001).

[4] P. Barbara et al., Phys. Rev. Lett. 82, 1963 (1999).

[5] I. Z. Kiss, Y. M. Zhai, and J. L. Hudson, Science 296, 1676 (2002); Phys. Rev. Lett. 94, 248301 (2005).

[6] Y. Kuramoto and I. Nishikawa, J. Stat. Phys. 49, 569 (1987).

[7] G.B. Ermentrout and J. Rinzel, J. Math. Biol. 22, 1 (1985); H. Daido, Prog. Theor. Phys. 75, 1460 (1986); ibid. 77, 622 (1987); H. Hong et al., Phys. Rev. E 59, 353 (1999).

[8] S. H. Strogatz, Physica D 143, 1 (2000).

[9] For a recent review, see J. A. Acebrón et al., Rev. Mod. Phys. 77, 137 (2005); and references therein.

[10] M. C. Cross and P. C. Hohenberg, Rev. Mod. Phys. 65, 851 (1993).

[11] G. Grinstein et al., Phys. Rev. Lett. 70, 3607 (1993); H. Chaté, G. Grinstein, and L.-H. Tang, ibid. 74, 912 (1995).

[12] S. De Monte et al., Phys. Rev. Lett. 92, 254101 (2004); Physica D 205, 25 (2005); Prog. Theor. Phys. Suppl. 161, 27 (2006).

[13] See, e.g., M. Barahona and L. M. Pecora, Phys. Rev. Lett. 89, 054101 (2002); M. Timme, F. Wolf, and T. Geisel, ibid. 89, 258701 (2002); T. Nishikawa et al., ibid. 91, 014101 (2003); H. Hong et al., Phys. Rev. E 69, 067105 (2004); C. Zhou et al., Phys. Rev. Lett. 97, 238103 (2006).

[14] H. Sakaguchi, S. Shinomoto, and Y. Kuramoto, Prog. Theor. Phys. 77, 1005 (1987).

[15] H. Daido, Phys. Rev. Lett. 61, 231 (1988).

[16] S. H. Strogatz and R. E. Mirollo, J. Phys. A 21, L699 (1988); ibid. 21, 4649 (1988).

[17] H. Hong, H. Park, and M. Y. Choi, Phys. Rev. E 70, 045204(R) (2004); ibid. 72, 036217 (2005).

[18] R. Botet, R. Jullien, and P. Pfeuty, Phys. Rev. Lett. 49, 478 (1982). 
[19] H. Daido, J. Stat. Phys. 60, 753 (1990). In the globally coupled case, $\gamma$ depends sensitively on how the oscillator frequencies are drawn (H. Hong et al., unpublished). 